\title{
Agarose hydrogel microcompartments for imaging sleep- and wake-like behavior and nervous system development in Caenorhabditis elegans larvae
}

\author{
Henrik Bringmann* \\ Max Planck Institute for Biophysical Chemistry, Am Fassberg 11, 37077 Göttingen, Germany
}

\section{A R T I C L E I N F O}

\section{Article history:}

Received 16 May 2011

Accepted 13 July 2011

\section{Keywords:}

C. elegans

Microfluidics

Sleep

\begin{abstract}
A B S T R A C T
Caenorhabditis elegans larvae display specific behavior and development that is not observed in adults. For example, larvae go through a molting cycle that includes a sleep-like state prior to the molt. The study of these processes requires high-resolution long-term observation of individual animals. Here we describe a method for simultaneous culture and observation of several individual young $C$. elegans larvae inside agarose hydrogel-based arrayed microcompartments. We used agarose hydrogel microcompartments to observe and quantify larval specific sleep-wake-like behavior and to observe neuronal rewiring using confocal fluorescence microscopy without acute immobilization. We found no behavioral aberrations caused by area restriction. We show that worms cultured inside hydrogel microcompartments develop into normal adults. Thus, hydrogel microcompartments appear useful for long-term observation of larval behavior and development.
\end{abstract}

() 2011 Elsevier B.V. All rights reserved.

\section{Introduction}

Caenorhabditis elegans is an established model system to study behavior and nervous system development. Most behavioral studies of C. elegans focus on adult animals, whereas comparatively little is known about larvae. However, C. elegans larval biology is extremely interesting and several features, such as a developmentally controlled sleep-wake cycle that is coupled to the molting cycle, occur only in larvae.

C. elegans larvae go through four larval stages termed L1-L4. At the end of each larval stage, the animals molt. C. elegans larval activity largely consists of feeding and concomitant body movements. C. elegans feeds on bacteria. A rhythmically contracting pharynx muscle pumps bacteria through a mouth in the tip of the head into the intestine. During feeding, animals typically display a dorsalventral rhythmic head movement called "foraging." Additionally, $C$. elegans displays hole-body locomotion (Riddle et al., 1997). Locomotion is reduced in the presence of bacterial food (Sawin et al., 2000). Another type of body movement are left right turns. On flat surfaces, animals lie either on their left or their right side. A movement initiated in the head can cause a "flip" to the other side. These left-right turns typically occur in larvae prior to the molt (Singh and Sulston, 1987).

\footnotetext{
* Corresponding author. Tel.: +49 $05512011358 / 2011091$; fax: +49 05512011092.

E-mail address: Henrik.Bringmann@mpibpc.mpg.de
}

Larvae are active most of each developmental stage, except during a period of behavioral quiescence called "Lethargus" preceding each molt. During Lethargus, animals stop feeding and greatly reduce body movements. Pharyngeal pumping and body movements resume just before the molt (Cassada and Russell, 1975).

Behavioral quiescence during Lethargus has been shown to be a sleep-like state: animals have an increased response threshold to external stimuli. Quiescence is homeostatically regulated. Lethargus is accompanied by molecular changes of genes homologous to genes that regulate circadian rhythms in other organisms (Raizen et al., 2008).

Not only the behavior of $C$. elegans larvae is interesting. Also certain developmental changes have only been observed in larvae and not in adults. For instance, in $C$. elegans larvae, new neurons are born and existing circuits rewire. An example for such a developmental change is the rewiring of the DD Neurons. The DD neurons are GABAergic motorneurons that form synapses with ventral body muscles in young L1 larvae. The DD neurons rewire starting at the end to the L1 stage. The DD neurons form new synapses with dorsal muscles and remove old synapses from ventral body wall muscles (Hallam and Jin, 1998; White et al., 1978).

While larval $C$. elegans display biological phenomena worth investigating, working with young $C$. elegans larvae is often difficult due to the small size of the animals. Observation of developmental changes requires continuous observation of individual animals by DIC and fluorescence microscopy. A classic technique for such studies involves mounting a larva and bacterial food between an agarose hydrogel pad and a coverslip. Animals usually stay near the food and their development can be followed by high-resolution 
DIC microscopy (Sulston, 1976). This technique has enabled many important studies of $C$. elegans development, such as the description of the entire postembryonic cell lineage of $C$. elegans (Sulston and Horvitz, 1977).

Typically, only one animal is mounted per sample to ensure that the same individual animal is repeatedly observed and the animal is tracked manually. Thus, this technique is time-consuming and has not been used for parallel processing of several animals using automated microscopes.

In the recent past microfluidic devices have been developed to facilitate the study of C. elegans and other small multicellular organisms (Chronis, 2010; Crane et al., 2010). C. elegans has been kept and imaged inside microcompartments for several days. Microcompartments have been made from PDMS (Hulme et al., 2010; Krajniak and Lu, 2010) or can be droplet-based (Shi et al., 2008). Most C. elegans devices have been optimized for adult animals, which are substantially larger than larvae.

Here we present a microfluidic device based on the classic agarose hydrogel mounting method. Young $C$. elegans larvae are cultured inside microcompartments made from agarose hydrogel filled with a thick bacterial suspension. Animals can be automatically imaged in physiological states using DIC and confocal microscopy. This microfluidic device allows confocal imaging without acute immobilization.

\section{Materials and methods}

\subsection{Agarose hydrogel microcompartment construction}

A detailed step-by-step protocol describing the technique including illustrations is available as a Supplementary file. Arrays consisted of $45 \times 45$ agarose microcompartments for small compartment size and $16 \times 16$ for big compartment size. One individual microcompartment was $190 \times 190 \times 10 \mu \mathrm{m}$ (height $\times$ width $\times$ depth) for small compartment size and $370 \mu \mathrm{m} \times 370 \mu \mathrm{m} \times 10 \mu \mathrm{m}$ for big compartment size. A spacer of $90 \mu \mathrm{m}$ separated individual compartments for small compartment size and a spacer of $320 \mu \mathrm{m}$ was used for big compartment size. Arrays of agarose hydrogel microcompartments were cast using 3-5 mm thick PDMS slabs containing the molds. PDMS molds were fabricated at Stanford Microfluidics Foundry using standard hard and soft lithography procedures. The side of the PDMS slabs that was opposite the side that contained the molds was bonded to a glass slide after exposure to air plasma for $60 \mathrm{~s}$ (Harrick Plasma, 0.5 mbar, settings to "medium"). The mold surface was then made hydrophilic by exposure to air plasma for $60 \mathrm{~s}$ as above. Molds were reused about 30 times and were cleaned using distilled water. After drying molds were treated with air plasma for $60 \mathrm{~s}$ directly before each use. We made two spacers out of stacks of glass slides glued together with double-sided sticky tape and placed the glass slide containing the mold with the PDMS slab and the side containing the molds downwards onto the spacers so that the mold surface did not touch the ground. The height of the stacks was adjusted so that a glass slide fitted underneath the mold surface leaving a gap between mold surface and glass slide of 1-1.5 mm. The glass slide was heated to $95^{\circ} \mathrm{C}$ on a heating block. $3 \%$ high melting point agarose (Fisher Bioreagents, BP(E)164-1000) in S-Basal (Stiernagle, 2006) was solved and stored in $2 \mathrm{ml}$ Eppendorf tubes as a stock and was melted just before use using a heating block at $95^{\circ} \mathrm{C}$. We placed $1 \mathrm{ml}$ of hot agarose onto the heated glass slide and moved the PDMS mold horizontally into the liquid agarose. After solidifying for $2 \mathrm{~min}$, we removed the PDMS molt and immediately filled the microcompartments with Escherchia coli bacteria and $C$. elegans eggs using a platinum wire pick. Both E. coli OP50 bacteria and C. elegans were grown on NGM plates as described (Brenner,
1974). Excess agarose hydrogel was then cut off using a scalpel. Using forceps we placed the square agarose hydrogel slab onto a $22 \mathrm{~mm} \times 22 \mathrm{~mm}$ coverslip with the microcompartment side facing the coverslip. This sealed the compartments. We used double-sided sticky tape to glue the coverslip containing the agarose hydrogel slab into a $20 \mathrm{~mm} \times 20 \mathrm{~mm}$ opening cut into the bottom of a $3.5 \mathrm{~cm}$ plastic Petri dish that was already prefilled with agarose hydrogel. To prepare the Petri dish, we first cut the $20 \mathrm{~mm} \times 20 \mathrm{~mm}$ opening into the bottom of the plastic Petri dish. The dish was cleaned with water and ethanol before use. A $22 \mathrm{~mm} \times 22 \mathrm{~mm}$ piece of double-sided sticky tape was then placed symmetrically over the opening and thus closing it. The opening was then cut open again using a scalpel leaving a $2 \mathrm{~mm}$ sticky rim around the sides of the opening. The Petri dish was then filled with $2 \mathrm{ml}$ of $95^{\circ} \mathrm{C} 3 \%$ high melting point agarose in S-Basal (Stiernagle, 2006). We let the Petri dish cool down before further use. After gluing the coverslip, which contained the agarose hydrogel slab, into the Petri dish the gap between the agarose slab containing the microcompartments and agarose filling the Petri dish was filled with 3\% low melting point agarose in S-Basal cooled to approximately $30^{\circ} \mathrm{C}$. After solidification of the agarose hydrogel, a custom-made aluminum lid was placed onto the Petri dish. The lid firmly closed the Petri dish to prevent drying of the agarose. The microcompartments were thus kept in an air reservoir. The lid contained a round $30 \mathrm{~mm}$ window made from $1.5 \mathrm{~mm}$ thick glass to allow DIC microscopy. The lid was heated to $25.5^{\circ} \mathrm{C}$ using resistors. Room temperature was kept at $20-21^{\circ} \mathrm{C}$. Sample temperature was measured using a thermo element and was around 23 or $24.5^{\circ} \mathrm{C}$ using a $20 \times$ dry or $40 \times$ oil objective, respectively. The agarose hydrogel was thus kept cooler than the lid, which prevented fogging of the glass window and drying of the agarose hydrogel.

\subsection{Imaging}

All imaging was performed on a Nikon TiE microscope controlled by iQ software (Andor). Focus was kept constant using Nikon "Perfect Focus System." Multifield imaging was done using a ProScan II (Prior Scientific) automated $x y$ stage set to lowest possible acceleration speed. DIC imaging was done using light of a 100 W Halogen lamp filtered though a D660/50 m filter (Chroma) to obtain red light. For DIC microscopy, animals were constantly illuminated. A $20 \times$ CFI Planapochromat VC Objective (Nikon) and a cooled Qicam camera (Qimaging) were used for DIC microscopy. Exposure time was $5 \mathrm{~ms}$. Spinning disc confocal imaging was done with an 'Andor Revolution' system using $488 \mathrm{~nm}$ Laser excitation and an iXon DU-897-BV EMCCD camera. A standard GFP emission filter (ET525/50 m, Chroma) was used. Exposure time was $15 \mathrm{~ms}$. Stacks of 15 frames each were taken using a "Nano Scan $Z$ " (Prior Scientific). $Z$-resolution of the stacks was $1 \mu \mathrm{m}$. Stacks were projected into one image using maximum pixel intensity. Under these conditions, only about every 4th frame showed signs of blurring during the wake-like state. During the sleep-like state, blurring was never observed. Thus, sufficient frames can be taken to follow rewiring. DD synapses were visualized using juIs137 [pflp-13::snb-1::GFP] (Baran et al., 2010).

\subsection{Analysis of adult C. elegans after culture in microcompartments}

To recover worms at the L2 stage the agarose hydrogel slab containing the microcompartments was cut out with a scalpel and transferred to an NGM plate seeded with $E$. coli OP50. A drop of $\mathrm{S}$ Basal $(0.5 \mathrm{ml})$ was placed onto the coverslip glass surface just after removal of the agarose slab. Most worms (approximately 80\%) stuck to the agarose pad, the remaining worms were found swimming in the $S$ Basal. Individual worms stuck to the agarose slab 

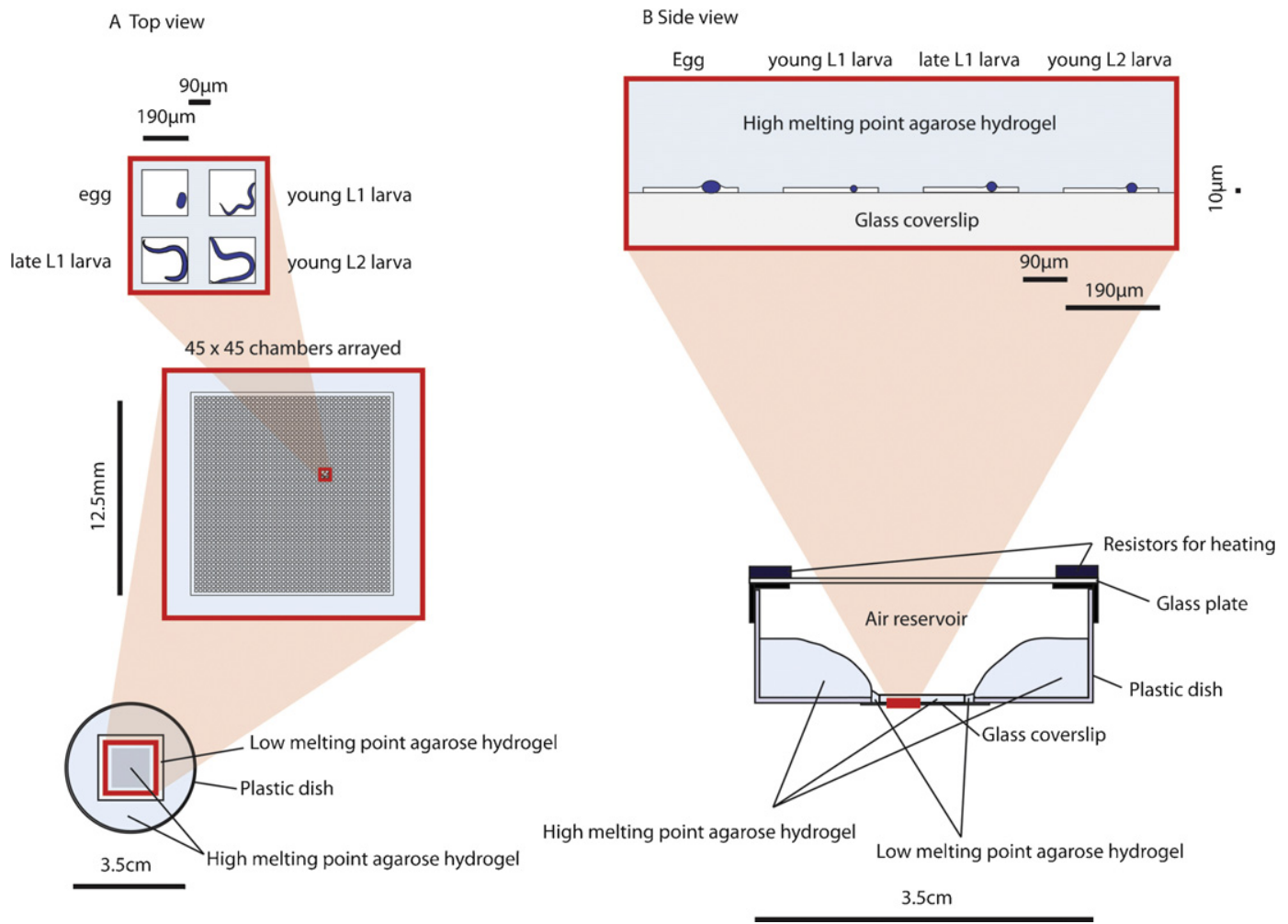

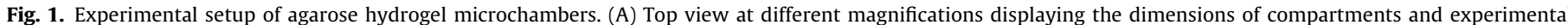

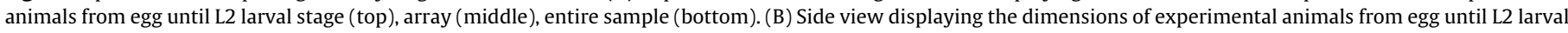
stage (top) and entire sample (bottom). Note that animal diameter is always smaller than compartment depth allowing confinement to the focal plane.

were transferred with a platinum wire pick off the agarose slab onto individual NGM plates seeded with E. coli OP50. Individual worms swimming in the drop of S Basal were recovered using a glass pipette drawn from a small glass capillary and transferred onto individual NGM plates seeded with E. coli OP50. We cultured control animals by placing eggs onto NGM plates (one egg per plate). We transferred the eggs just after filling the chamber. Both control worms and microcompartment worms were kept at $20^{\circ} \mathrm{C}$ in the same room. After rescue of L2 larvae both rescued worms and control worms were placed into a $20^{\circ} \mathrm{C}$ incubator for 3 days. During this time, worms were visually inspected every $12 \mathrm{~h}$ using a stereomicroscope. One hour prior to the tap experiment, all worms were transferred to fresh NGM plates using a platinum wire pick. We filmed worms with a rate of $10 \mathrm{frames} / \mathrm{s}$ and delivered five taps using a function generator to trigger a metal piston driven by an electromagnet (Kuhnke, H22, 24 V, tap duration $200 \mathrm{~ms}$, taps spaced $500 \mathrm{~ms}$ ). The electromagnet was fixed to the dish containing the worms by a homemade aluminum frame that ensured that the piston hit the dish horizontally.

\section{Results}

\subsection{Culturing young C. elegans larvae in agarose hydrogel microcompartments}

We constructed arrays of shallow microcompartments from agarose hydrogel using a polydimethylsiloxan (PDMS) mold (see Section 2.1, Fig. 1, and Supplementary protocol). To keep the animals in the field of view the $x y$ dimensions of the individual chamber were chosen to almost fill the field of view of the camera, here $190 \mu \mathrm{m} \times 190 \mu \mathrm{m}$. To keep the animals in the focal plane, the depth of the compartments was chosen to be $10 \mu \mathrm{m}$. The depth of the compartment is thus smaller than the diameter of the smallest
C. elegans, which is a freshly hatched C. elegans L1 larva (about $12-13 \mu \mathrm{m}$ in diameter). This means that the animal was partly pressed into the soft agarose hydrogel and was thus kept in one focal plane.

One side of the hydrogel chambers was left open to allow filling of the compartment. We filled compartments manually with one C. elegans egg and E. coli bacteria as a food source. The chambers were then closed with a coverslip and placed into a temperaturecontrolled humidified chamber (see Section 2.1 and Fig. 1). The microcompartments were placed onto an inverted microscope and a time-lapse movie of a single animal was taken with one frame every $5 \mathrm{~s}(N=6)$. As shown in Fig. 2 , the interface between agarose hydrogel and glass coverslip formed a tight seal that prevented the animals from escaping the chamber until at least the L2 stage. The animals were confined to the focal plane as expected. Animals hatched without any apparent problem inside the compartments and developed at least to the end of the L2 stage. Ample food was present at least until the L2 stage. Thus, agarose hydrogel microcompartments can be used to confine young C. elegans larvae to the field of view and focal plane of the camera chip during long-term observations.

\subsection{Behavior of C. elegans inside agarose hydrogel microcompartments}

To probe the usefulness of our devices we tested whether C. elegans displays typical larval behavior, such as sleep and wake-like behavior inside agarose hydrogel microcompartments. We quantified movement, feeding, and left-right turning of $C$. elegans larvae that were cultured inside the compartments.

We first quantified movement by manually tracking the tip of the head (Fig. 3). Animals displayed continuous head movement during the entire L1 wake-like period. However, head movement 


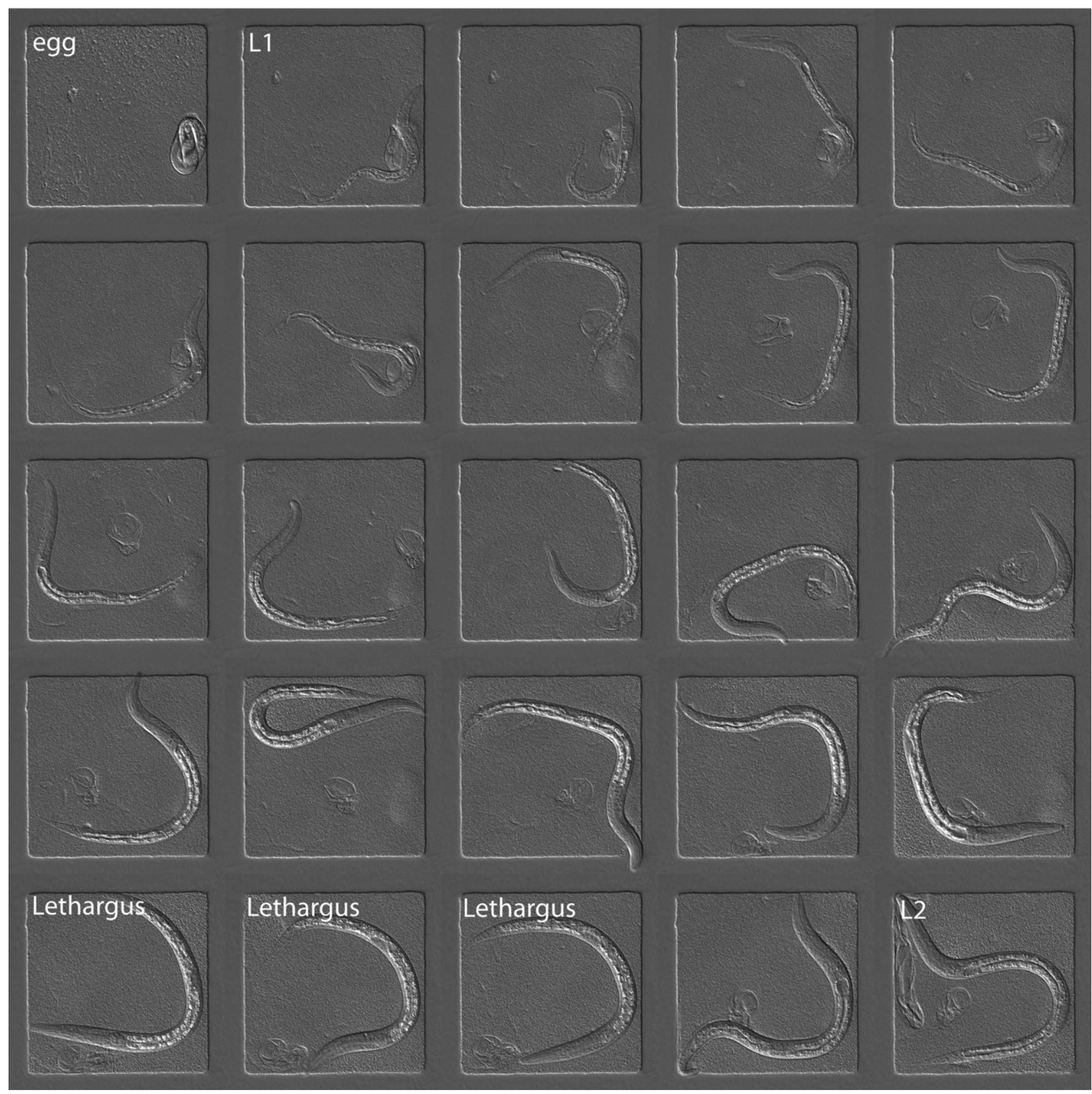

Fig. 2. Agarose hydrogel microcompartments can be used for DIC observation of C. elegans from egg until L2 larval stage. Displayed is every 400 th frame of a 5-s interval timelapse movie. The first frame shows an egg just before hatching. Frame 2 shows an early L1 animal. Frames labeled Lethargus show the animal in the L1 sleep-like state. Note the elongated, relaxed body posture and the lack of large-scale movements. Frame labeled L2 shows an animal just after the first molt. Note the shed cuticle in this last frame. Compartment dimensions were $190 \mu \mathrm{m} \times 190 \mu \mathrm{m}$. Compartments were $10 \mu \mathrm{m}$ deep.

was strongly reduced in a period starting $1.5-2 \mathrm{~h}$ before and ending 15-20 min before the molt. During this time, animals displayed periods of total immobility, not seen during the rest of L1. Head movements caused by twitching of the worm interrupted the quiescence. We observed no large-scale locomotion during this time. This quiescent period coincided with the absence of feeding, which can be scored by the shape and appearance of the pharynx muscle. Total nose immobility lasting at least $5 \mathrm{~s}$ during the nonfeeding period was $27 \pm 2 \min (N=6, S E M)$.

The $C$. elegans pharynx pumps several times per second during feeding. To precisely measure pumping rates, we took short movies of animals every $10 \mathrm{~min}$. Short movies consisted of 100 frames taken within $4.5 \mathrm{~s}$. Several microcompartments containing one animal each were observed in parallel by using a slowly moving automated scanning stage that consecutively moved the microcompartments into the field of view of the camera chip. We looked at the grinder and counted one cycle of contraction and relaxation of the terminal bulb of the pharynx as one pump. Pumping is displayed in Fig. 4. Freshly hatched L1 animals had a relatively low pumping frequency. As the animals mature, pumping frequency increased until a plateau was reached. A period of continuous absence of pumping preceded the molt. When entering the nonpumping phase, animals rapidly decease pumping speed within minutes. When exiting the nonpumping phase, animals resume pumping and accelerate pumping frequencies within minutes to levels before the nonpumping phase. The average time during which no pumping was observed was $1 \mathrm{~h} 46 \mathrm{~min} \pm 4 \mathrm{~min}$ (SEM, $N=5)$.

We scored left-right turns manually from our 5-s interval timelapse movies. Fig. 3 shows left-right turn occurrence for one animal. 


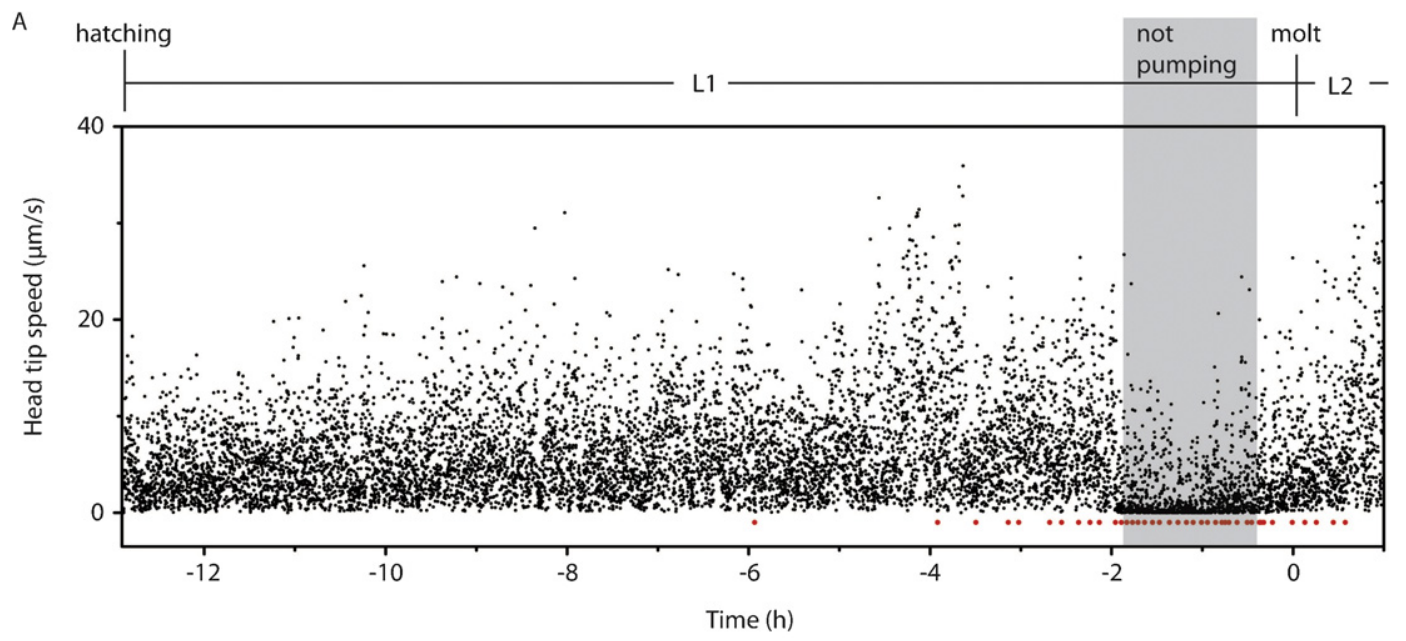

B
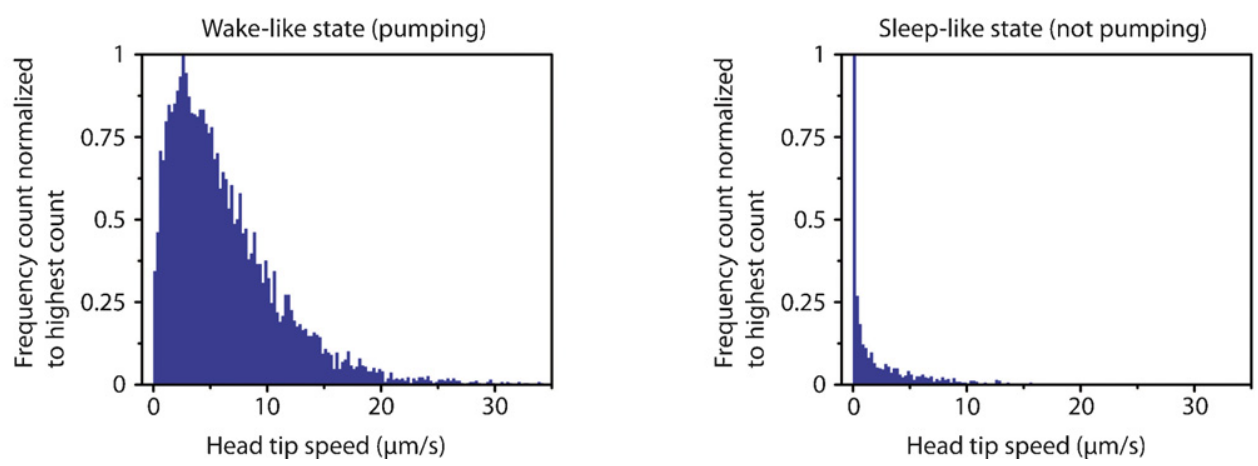

Fig. 3. Agarose hydrogel microcompartments can be used to quantify movement during larval sleep and wake-like behavior. (A) Speed of the tip of the head versus time from hatching until early L2 stage (black points). Time 0 corresponds to completion of the molt. Nonfeeding period is displayed in grey. Left-right turns are displayed as red points. (B) Frequency count of head tip speed during L1 for the wake-like (left) and sleep-like/nonfeeding (right) state. Frame bin was $0.25 \mu \mathrm{m} / \mathrm{s}$. Data was normalized to highest count. (For interpretation of the references to color in this figure legend, the reader is referred to the web version of the article.)

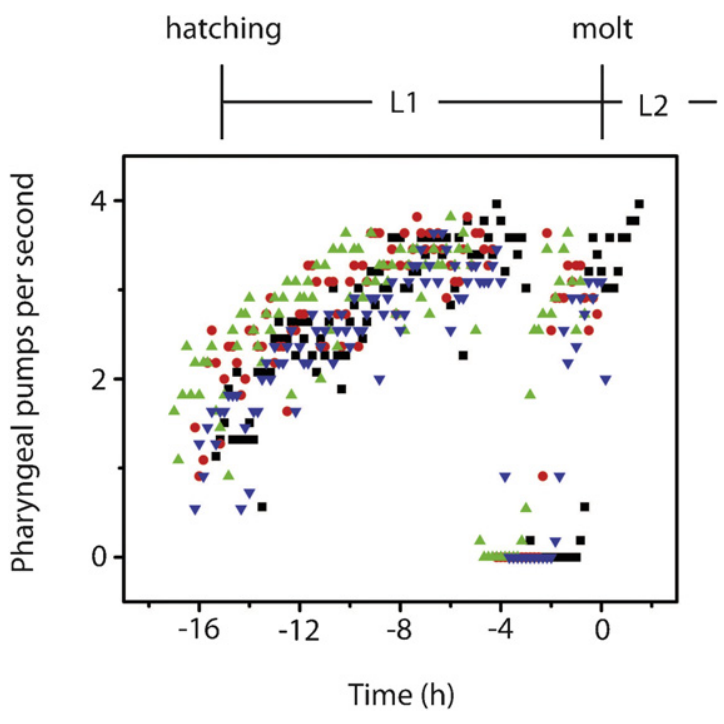

Fig. 4. Agarose hydrogel microcompartments can be used to quantify pharyngeal pumping during larval sleep and wake-like states. Colors represent four individual animals. Time 0 corresponds to completion of the first molt. Note the increase of pumping in the course of the L1 wake-like phase and the complete absence of pumping preceding the molt. (For interpretation of the references to color in this figure legend, the reader is referred to the web version of the article.)
Each left-right turn is displayed as one red dot. Left-right turns occurred predominantly towards the end of the larval cycle preceding the molt. However, left right turns were not strictly restricted to the nonpumping and nonmoving period but extended until the actual molt and were sometimes observed up to $1 \mathrm{~h}$ after the molt. Animals turned on average $23 \pm 3$ times during the entire L1 stage. $14 \pm 1$ of these turns occurred during the nonpumping period $(N=6$ worms, SEM).

These results show that animals display typical behavior inside agarose hydrogel microcompartments. For the first time, these C. elegans behaviors have been monitored automatically with high-resolution DIC microscopy using L1 larvae and have been quantified. Cessation of pumping and reduction of movement always coincide and appear to constitute the sleep-like state. As left-right turns do not coincide precisely with the period of quiescence, they may not be a consequence of the quiescence but rather serve a more direct role in molting.

\subsection{Effects of area restriction on behavior}

We have developed microcompartments to restrict large-scale movement of $C$. elegans, allowing long-term observation of several animals simultaneously. How does restriction of $C$. elegans larvae to small areas affect behavioral parameters? We have constructed microcompartments that provide approximately four times more space $(370 \mu \mathrm{m} \times 370 \mu \mathrm{m} \times 10 \mu \mathrm{m})$. We have cultured and filmed C. elegans larvae in these bigger compartments and have compared their behavior with worms cultured inside smaller compartments $(190 \mu \mathrm{m} \times 190 \mu \mathrm{m} \times 10 \mu \mathrm{m})$ (Fig. 5). 


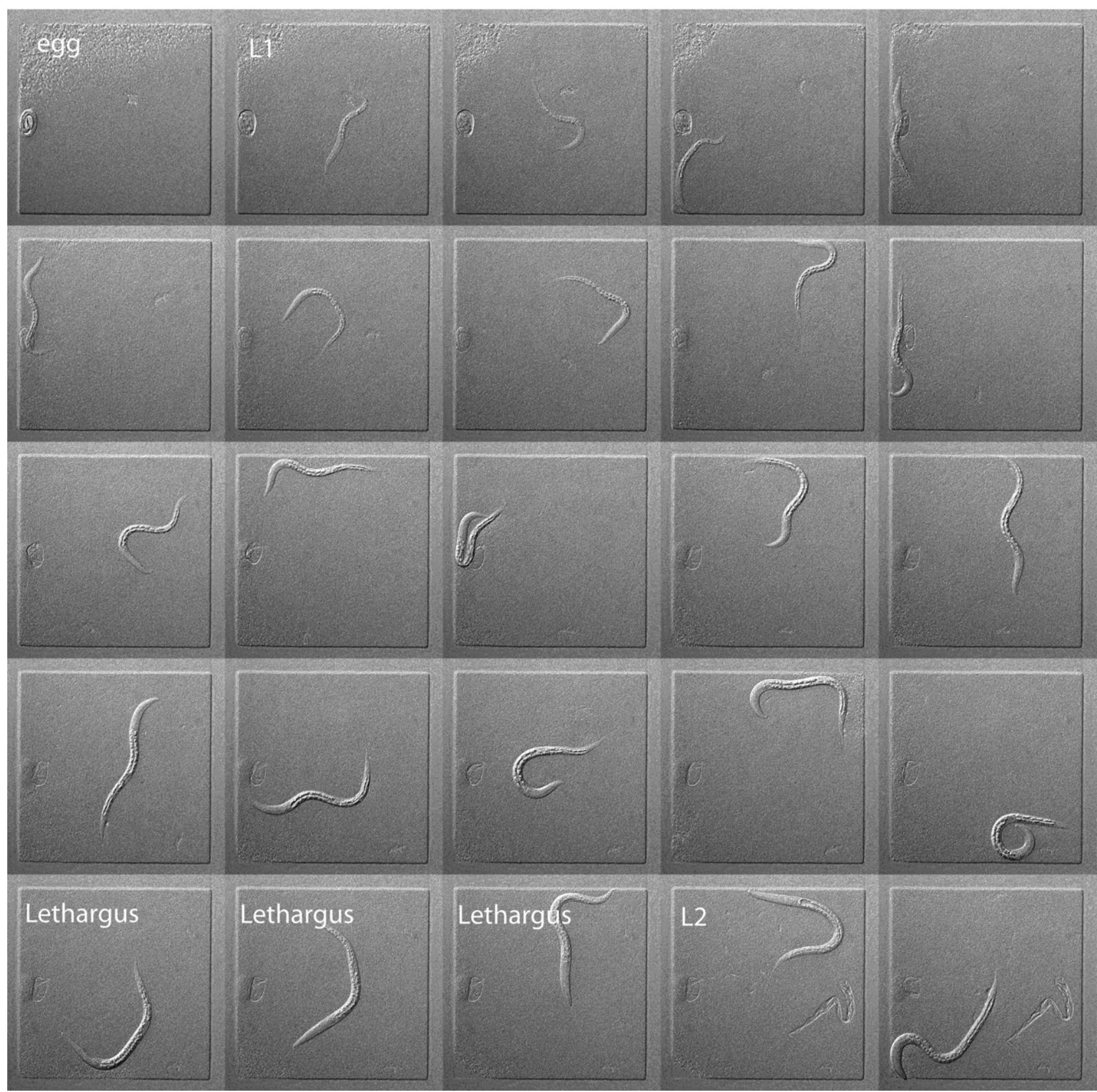

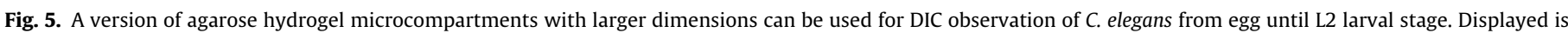

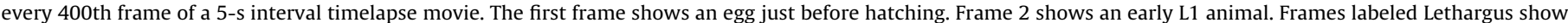

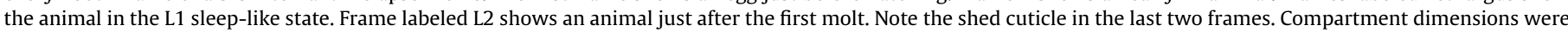
$370 \times 370 \mu \mathrm{m}$. Compartments were $10 \mu \mathrm{m}$ deep.

We first looked at the relative position of the worm nose inside the compartments (Fig. 6A). Inside the smaller compartment, the worm nose appeared at the border and corners of the compartment much more often compared with the bigger compartment. We then looked at the speed and directionality of worm movement. Worm movement in smaller compartments was similar to movement in bigger compartments. In both types of compartments, worms preferentially moved forward during $57 \%$ of the time during the wake-like state (Fig. 6B).

The results indicate that compartment area size does not grossly influence worm movement. Agarose microcompartments restrict long-range movement by providing a mechanical barrier that the worms cannot overcome and not by decreasing movement. Consistent with this observation worms often move forward inside the compartment until they reach the border and then keep moving along the border. In smaller compartments, increased touching of the compartment border by the nose does not appear to cause gross reduction of movement and does not appear to cause direction reversals.

\subsection{Effects of microcompartment culture on survival}

We have not observed any animal to die during the confinement period in the above experiments. How does microcompartment culture affect survival after the confinement? To address this question we cultured $C$. elegans larvae inside microcompartments from pretzel stage egg until the L2 larval stage and then moved the worms onto normal NGM plates (see Section 2). All individual C. 


\section{A nose position during the L1 wake-like state (black) and sleep-like state (red)}

\section{$190 \mu \mathrm{m} \times 190 \mu \mathrm{m} \times 10 \mu \mathrm{m}$}

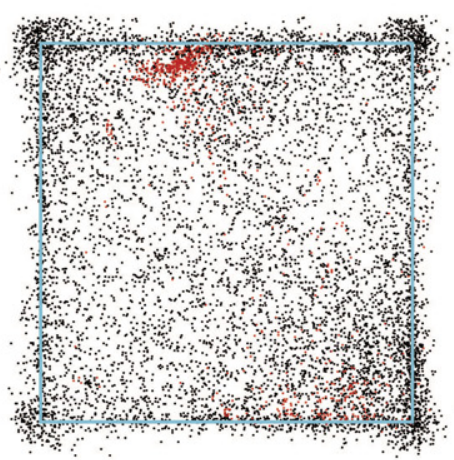

\section{$370 \mu \mathrm{m} \times 370 \mu \mathrm{m} \times 10 \mu \mathrm{m}$}

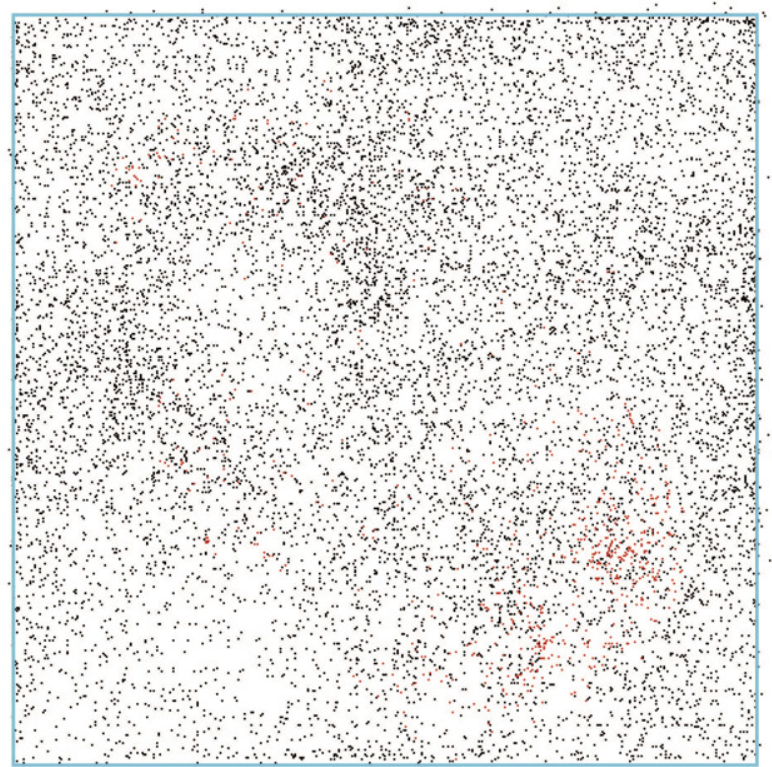

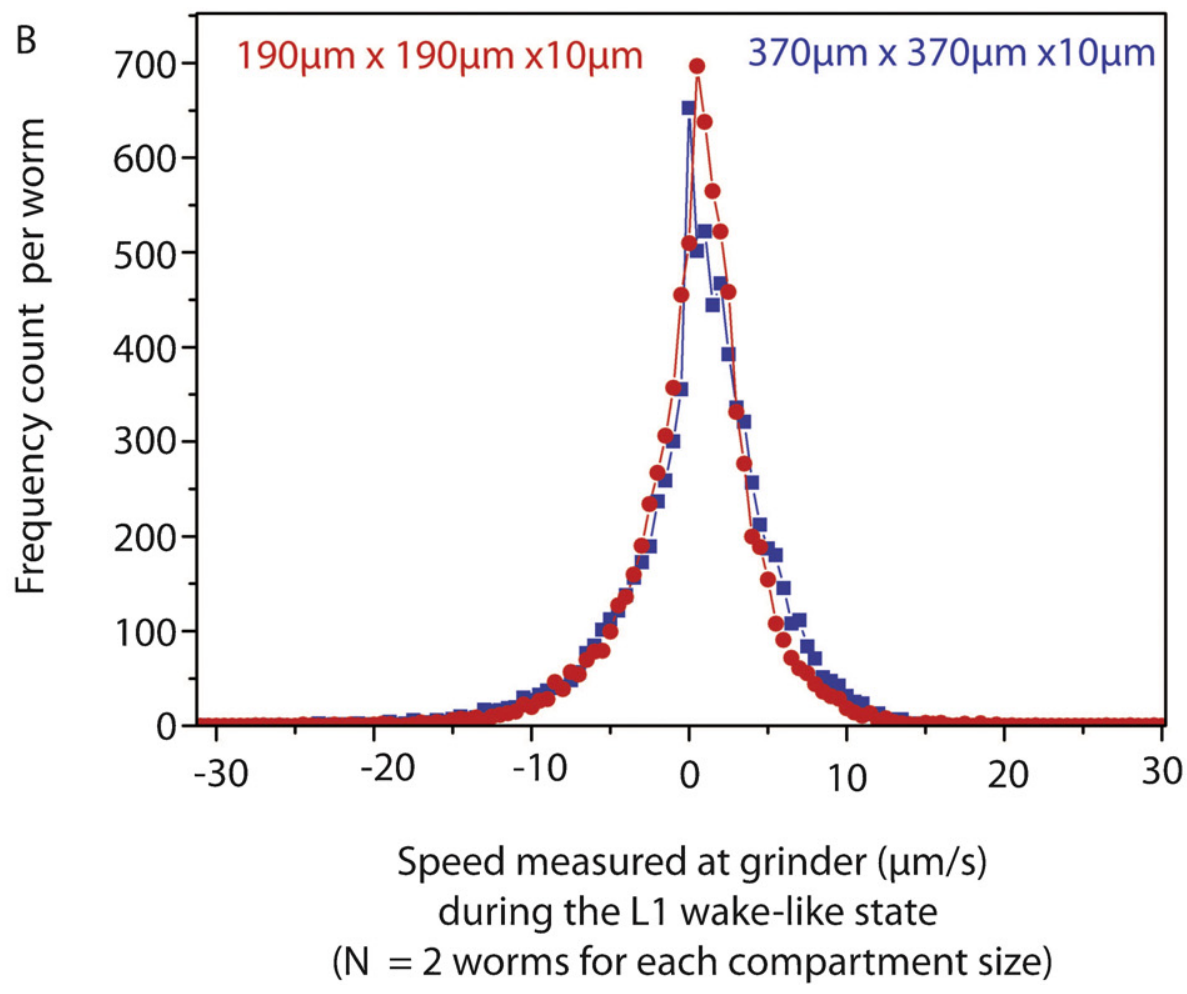

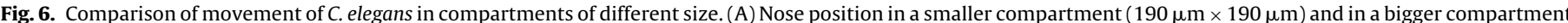

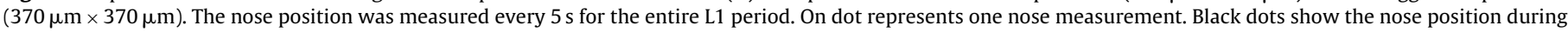

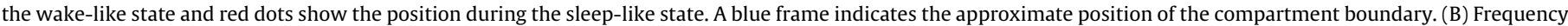

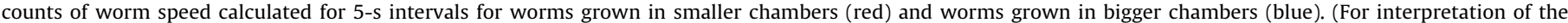
references to color in this figure legend, the reader is referred to the web version of the article.)

elegans could be recovered from the compartment (75/75), and all animals (75/75) developed directly into fertile adults without passing through a dauer stage. We thus conclude that culture conditions inside agarose hydrogel microcompartments do not interfere with normal C. elegans development.

\subsection{Effects of microcompartment culture on adult behavior}

Animals cultured inside microcompartments developed into healthy adults. Inside microcompartments animals constantly touch the agarose and glass surface - providing a mechanical 
stimulus that the $C$. elegans larvae may sense. How does this mechanical stimulation during culture affect adult behavior?

We looked at the dish tap reflex of adult $C$. elegans that had been cultured inside microcompartment from egg until L2 stage. We filmed the worms on NGM plates and we delivered a train of five taps using an electromagnetic tapper (see Section 2). Both, worms grown in "free range" and worms grown in microcompartments responded in all cases with a backwards movement lasting 2-5 s ( $N=5$ worms for each condition, Fig. 7$)$. We could thus not find any indication for an abnormal mechanosensory behavior of worms cultured inside agarose hydrogel microcompartments.

\subsection{Imaging of $D D$ neuron rewiring}

We next imaged nervous system rewiring in individual young $C$. elegans larvae. We used transgenic $C$. elegans that express SNB::GFP in the six DD neurons to visualize synapses (Baran et al., 2010). We filmed animals with a spinning disc confocal microscope. Fig. 8 shows selected frames that show the rewiring of the DD neurons. During early L1 (timepoints $-8: 40$ and $-1: 40$, h:min) only ventral SNB::GFP was visible. Dorsal DD SNB::GFP was first visible during L1 Lethargus in the anterior of the animal (timepoints -0:50 and $-0: 30)$. At that time ventral SNB::GFP was still prominently visible. During the L2 stage dorsal SNB::GFP increased in the anterior and also became visible in the posterior of the animal (timepoints $+0: 20$ to $+7: 20$, h:min). By the early L3 stage, dorsal SNB::GFP was visible along the entire dorsal cord (timepoint $+9: 20$ ). The signal was stronger towards the anterior of the animal. Reduction of ventral SNB::GFP was observed during the entire L2 period and is largely completed around the L3 stage as ventral SNB::GFP is barely visible at the L3 stage.

We thus conclude that agarose hydrogel microcompartments can be used for confocal observations of nervous system development. Our results indicate that DD neuron rewiring may include a bipolar intermediate in which both ventral and dorsal DD synapses exist. We observe dorsal synapses first during L1 Lethargus. Agarose hydrogel microcompartments can thus be used to correlate behavior and nervous system development. The slowing response of $C$. elegans in the presence of bacterial food and the confinement of the animals by the compartments may reduce movements to a level where acute immobilization of animals is not necessary for even confocal imaging.

\section{Discussion}

\subsection{An improved system for imaging C. elegans larvae}

Investigation of the nervous system using fluorescenc microscopy requires immobilization of the animal or short exposure time in the range of $5-15 \mathrm{~ms}$ to avoid blurring of the image due to animal movement. Such short exposure times can be achieved using EMCCD cameras and spinning disc confocal scanners. Confocal microscopy typically requires acquisition of $\mathrm{z}$ stacks through the entire animal, allowing projection or threedimensional reconstruction. Even with short exposure times a total acquisition time of a stack is in the range of $700 \mathrm{~ms}$ - enough time for an animal to move and cause image distortions.

In some PDMS devices, animals can be immobilized repeatedly to avoid image distortions. Immobilization has been achieved mechanically, thermally, or chemically using pressure (Hulme et al., 2007, 2010), gelation (Krajniak and Lu, 2010), low temperatures (Chung et al., 2008), or carbondioxide (Chokshi et al., 2009).

However, all current immobilization methods have the potential to acutely change the physiological state of the nervous system.
A

Adult C. elegans grown in "free range"

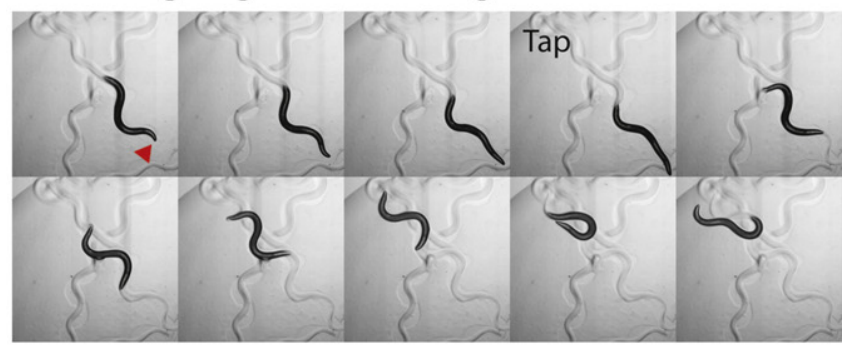

Adult C. elegans grown in microchamber until L2
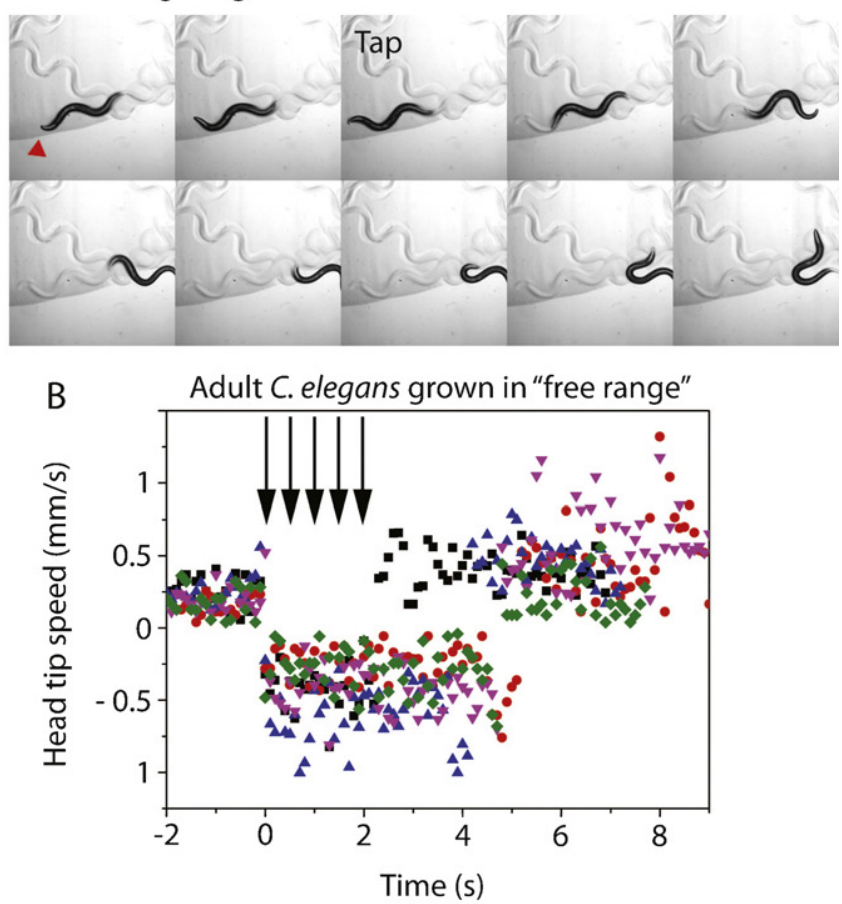

Adult C. elegans grown in microchamber until L2

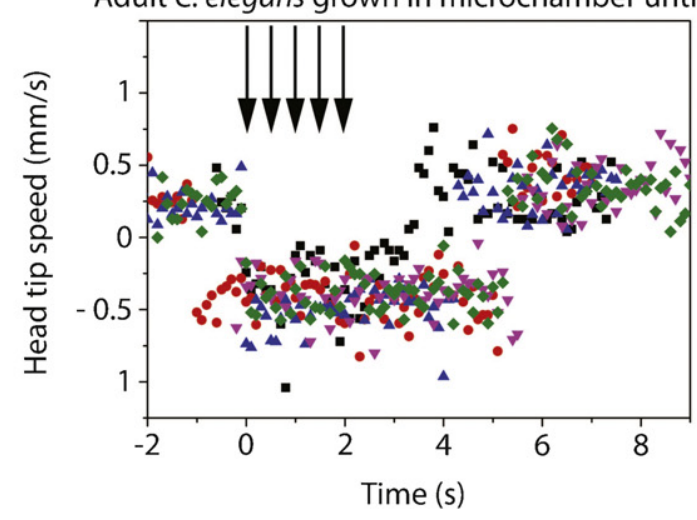

Fig. 7. C. elegans larvae cultured inside agarose hydrogel microcompartments develop into adults that show a normal behavioral response to dish tap. (A) Timelapse series of brightfield images of adult C. elegans on NGM plates during the tap experiment. The red arrow points to the head of the animal. Tapping start is indicated. The field of view displayed is approximately $2 \mathrm{~mm} \times 2 \mathrm{~mm}$. Images are $1 \mathrm{~s}$ apart. (B) Head tip speed versus time shown for five individual adult $C$. elegans. The times at which the taps were delivered to the worms is indicated by the black arrows. $N=5$ worms for each condition. Worms grown only on NGM plates reversed for $4.2 \pm 0.5 \mathrm{~s}$ (SEM, $N=5$ worms) with a speed of $-402 \pm 59 \mu \mathrm{m} / \mathrm{s}$ (SEM, $N=5$ worms). Worms grown in microcompartments during $\mathrm{L} 1$ reversed for $4.7 \pm 0.4 \mathrm{~s}$ (SEM, $N=5$ worms) with a speed of $-399 \pm 34 \mu \mathrm{m} / \mathrm{s}$ (SEM, $N=5$ worms). (For interpretation of the references to color in this figure legend, the reader is referred to the web version of the article.) 


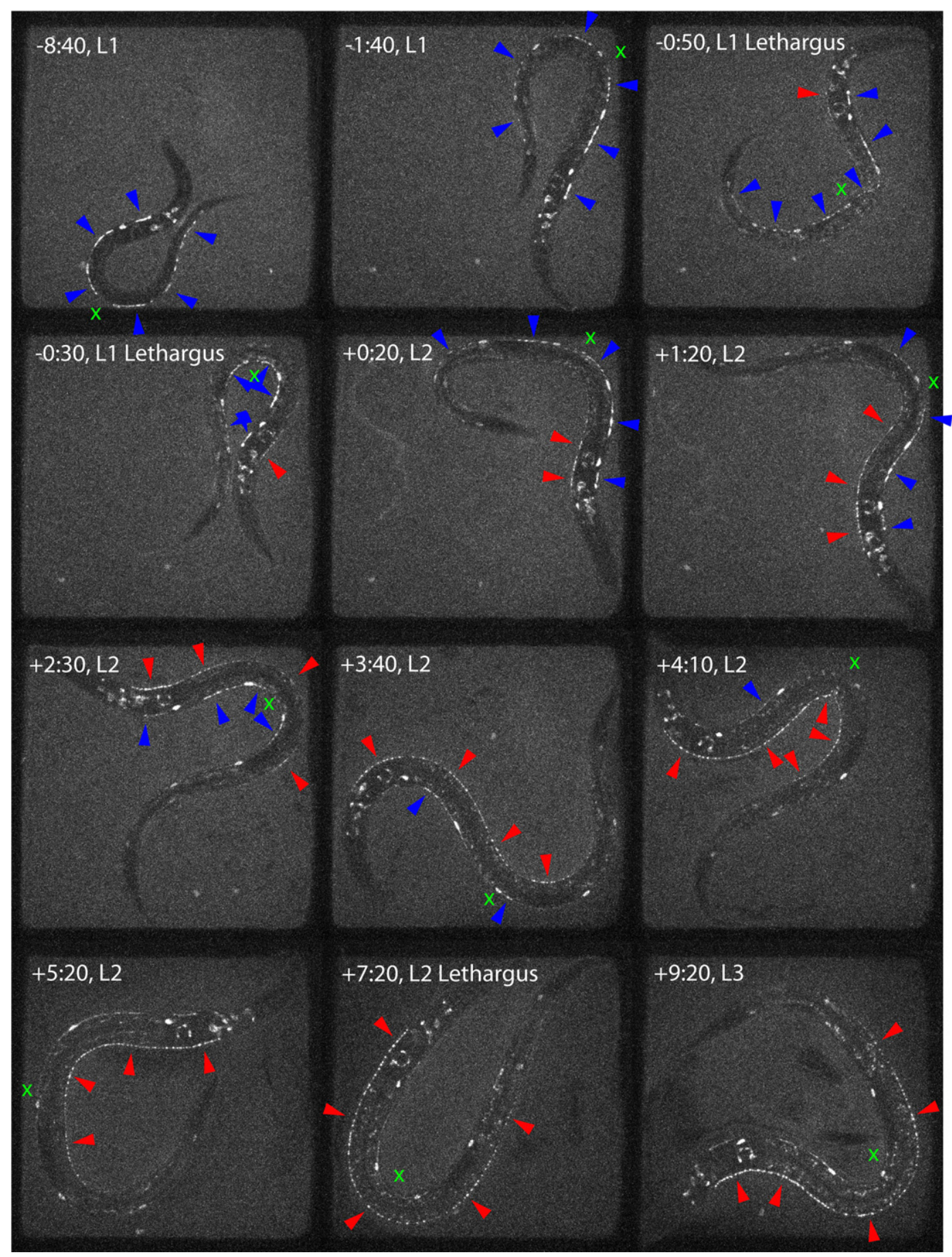

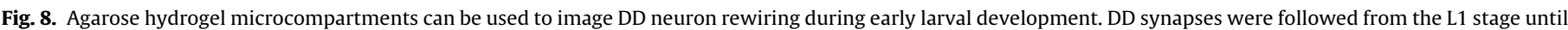

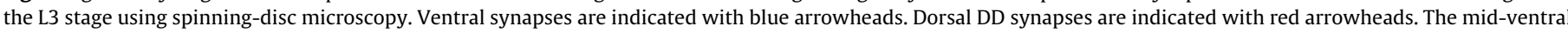

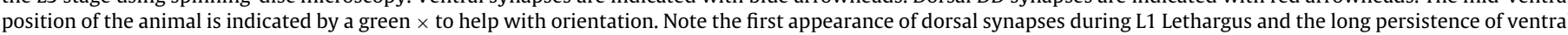

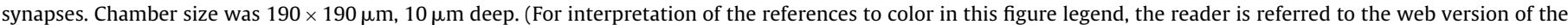
article.)

C. elegans has been shown to respond to mechanical stimulation, temperature changes, liquid chemicals and gases such as carbondioxide (Bargmann, 2006; Bretscher et al., 2008; Chalfie et al., 1985; Hallem and Sternberg, 2008).

Thus, it is useful to have a culture and imaging method that works without acute immobilization. An alternative to acute immobilization is to generally reduce animal movement to a level that is acceptable for imaging. Animal movement levels depend on confinement of the animal by the compartment and on endogenous animal movement. Endogenous movement cannot be fully avoided but varies greatly depending on environmental factors.

Z-movement in our compartments is restricted by compartment depth. Our compartments are only $10 \mu \mathrm{m}$ deep, deeper than any other published microcompartment system used for culture of $C$. elegans, and smaller than the smallest diameter of a newly hatched $C$. elegans larvae. As a consequence, the larva is always maximally confined to the $x$-y-plane minimizing the number of $z$-planes required per stack. Animals are kept in a thick bacterial 
paste. It has been shown that animals reduce their movement when they encounter a bacterial lawn on an agarose surface. This process is caused by mechanical stimulation of the animal by the bacteria (Sawin et al., 2000). Thus, an environment can be provided that delivers mechanical stimulation thus triggers reduced movement. The degree of movement reduction in our microcompartments appears sufficient for acquisition of stacks of confocal images without the need for acute immobilization.

\subsection{Optimization of compartment dimensions for specific experiments}

The smaller the area of a compartment is, the higher the resolution will be with which the worm can be imaged. The bigger the compartments area is the less restricted is long-range movement. The size of the microcompartment users should choose will depend on the scientific question and will always be a compromise between restricting behavior or resolution. We have found that $190 \times 190 \mu \mathrm{m}$ presents a good compromise if L1 behavior such as sleep-like states and neural development should be observed simultaneously. If only behavior needs to be observed, bigger compartments $(370 \mu \mathrm{m} \times 370 \mu \mathrm{m})$ are more comfortable and easy to use. If users wish to reduce confinement to one $\mathrm{z}$ plane, deeper compartments can be used. We have made compartments that are $15 \mu \mathrm{m}$ deep and allow culture and imaging similar to $10 \mu \mathrm{m}$ deep compartments. However, due to the reduced $z$ confinement image quality is slightly compromised with $15 \mu \mathrm{m}$ deep compartments (data not shown).

\subsection{Effects of compartment culture on behavior and development}

Disfavorable culture conditions may cause dauer formation or even death (Cassada and Russell, 1975). The decision to enter the dauer stage is made during the L1 stage and depends on several factors such as the availability of sufficient food (Golden and Riddle, 1984). In our culture system, larvae are kept inside microcompartments for the entire L1 larval stage. We have not observed dauer entry or death during or after microcompartment culture and thus conclude that our method provides rather favorable conditions for C. elegans.

Every culture method provides mechanical stimulation of the worms. Inside microcompartments, both left and right side of the worms constantly touch the compartment $z$ boundaries. The nose tip regularly touches the $x y$ boundaries.

Strong mechanical stimulation, such as nose touch with an eyelash, cause a withdrawal reflex (Chalfie and Sulston, 1981). When an eyelash is placed at $90^{\circ}$ into the path of a forward moving worm the animal will touch the eyelash with its nose, stop moving forward, and then initiate a backwards movement in $90 \%$ of collisions (Kaplan and Horvitz, 1993). Worms also respond to dish tapping by initiating a backwards response (Wicks et al., 1996).

Sensory stimulation of larvae has been shown to influence development and adult behavior. C. elegans is typically kept in culture together with other individuals. Individual animals regularly touch each other. Larvae can be deprived of this mechanical stimulation by raising them in isolation. Mechanically deprived worms show retarded development and reduced behavioral responses (Rose et al., 2005).

While strong mechanical stimuli cause avoidance reflexes, mild mechanical stimulation is important for worm development. We have assessed the nature of mechanical stimulation during microcompartment culture. We have looked at the behavior both inside the compartment as well as at the later adult stage. We found that increased nose-on collisions with the compartment border did not cause increased backwards movement. Thus, worms do not react to border collisions with an avoidance reflex. We found that worms cultured inside microcompartments responded as adults to strong dish taps by reversing movement similar to worms cultured on plates. Thus mechanical stimulation in our culture conditions is mild and rather favorable for $C$. elegans.

\section{Conclusion}

Almost all behavioral and developmental data on $C$. elegans has been collected with worms growing on an agarose hydrogel surface. We found that agarose hydrogel is an excellent material for constructing microcompartments for the culture of small C. elegans larvae. Our technique constitutes a link between classic observation techniques and recent silicon-based microfluidic devices. We have combined the well-investigated environment of the agarose hydrogel with microfluidics to follow the behavior of several individual animals in the same sample using automated microscopes. We showed that our culture method provides a favorable environment to the worms and that restricting worms to the compartments has no aversive effects on behavior. Agarose hydrogel has no toxic effects on C. elegans, can be easily cast with PDMS molds, can form tight seals with glass surfaces, and allows diffusion of nutrients, gases, and waste products. Thus, it is an ideal material for longterm studies of small C. elegans larvae, and potentially other motile microscopic organisms.

\section{Acknowledgements}

I thank Bill Schafer and Mario de Bono for hospitality during a stay at the MRC Laboratory for Molecular Biology where preliminary work was done, Stanford microfluidics foundry for device fabrication, Yishi Jin for juIs137, Hans-Dieter Scheede for building the lid heater. Hartmut Sebesse for the 3D drawings in the Supplementary protocol. Michael Pilot and Agnes Zarzycka for help with manual tracking.

\section{Appendix A. Supplementary data}

Supplementary data associated with this article can be found, in the online version, at doi:10.1016/j.jneumeth.2011.07.013.

\section{References}

Baran R, Castelblanco L, Tang G, Shapiro I, Goncharov A, Jin Y. Motor neuron synapse and axon defects in a C elegans alpha-tubulin mutant. PLoS One 2010;5:e9655. Bargmann CI. Chemosensation in C. elegans. WormBook 2006:1-29.

Brenner S. The genetics of Caenorhabditis elegans. Genetics 1974;77:71-94.

Bretscher AJ, Busch KE, de Bono M. A carbon dioxide avoidance behavior is integrated with responses to ambient oxygen and food in Caenorhabditis elegans. Proc Natl Acad Sci U S A 2008; 105:8044-9.

Cassada RC, Russell RL. The dauerlarva, a post-embryonic developmental variant of the nematode Caenorhabditis elegans. Dev Biol 1975;46:326-42.

Chalfie M, Sulston J. Developmental genetics of the mechanosensory neurons of Caenorhabditis elegans. Dev Biol 1981;82:358-70.

Chalfie M, Sulston JE, White JG, Southgate E, Thomson JN, Brenner S. The neural circuit for touch sensitivity in Caenorhabditis elegans. J Neurosci 1985;5:956-64.

Chokshi TV, Ben-Yakar A, Chronis N. CO2 and compressive immobilization of $C$. elegans on-chip. Lab Chip 2009;9:151-7.

Chronis N. Worm chips: microtools for C. elegans biology. Lab Chip 2010;10:432-7.

Chung K, Crane MM, Lu H. Automated on-chip rapid microscopy, phenotyping and sorting of C. elegans. Nat Methods 2008;5:637-43.

Crane MM, Chung K, Stirman J, Lu H. Microfluidics-enabled phenotyping, imaging and screening of multicellular organisms. Lab Chip 2010;10:1509-17.

Golden JW, Riddle DL. The Caenorhabditis elegans dauer larva: developmental effects of pheromone, food, and temperature. Dev Biol 1984;102:368-78.

Hallam SJ, Jin Y. lin-14 regulates the timing of synaptic remodelling in Caenorhabditis elegans. Nature 1998;395:78-82.

Hallem EA, Sternberg PW. Acute carbon dioxide avoidance in Caenorhabditis elegans. Proc Natl Acad Sci U S A 2008:105:8038-43.

Hulme SE, Shevkoplyas SS, Apfeld J, Fontana W, Whitesides GM. A microfabricated array of clamps for immobilizing and imaging C. elegans. Lab Chip 2007; 7:1515-23. 
Hulme SE, Shevkoplyas SS, McGuigan AP, Apfeld J, Fontana W, Whitesides GM. Lifespan-on-a-chip: microfluidic chambers for performing lifelong observation of C. elegans. Lab Chip 2010;10:589-97.

Kaplan JM, Horvitz HR. A dual mechanosensory and chemosensory neuron in Caenorhabditis elegans. Proc Natl Acad Sci U S A 1993;90:2227-31.

Krajniak J, Lu H. Long-term high-resolution imaging and culture of C. elegans in chip-gel hybrid microfluidic device for developmental studies. Lab Chip 2010;10:1862-8.

Raizen DM, Zimmerman JE, Maycock MH, Ta UD, You YJ, Sundaram MV, et al. Lethargus is a Caenorhabditis elegans sleep-like state. Nature 2008;451: 569-72.

Riddle DL, Blumenthal T, Meyer BJ, Priess JR. C. elegans II. 2nd ed. Cold Spring Harbor Mongraph Series; 1997. p. 33.

Rose JK, Sangha S, Rai S, Norman KR, Rankin $\mathrm{CH}$. Decreased sensory stimulation reduces behavioral responding, retards development, and alters neuronal connectivity in Caenorhabditis elegans. J Neurosci 2005;25: 7159-68.
Sawin ER, Ranganathan R, Horvitz HRC. elegans locomotory rate is modulated by the environment through a dopaminergic pathway and by experience through a serotonergic pathway. Neuron 2000;26:619-31.

Shi W, Qin J, Ye N, Lin B. Droplet-based microfluidic system for individual Caenorhabditis elegans assay. Lab Chip 2008;8:1432-5.

Singh RN, Sulston JE. Some observations on moulting in Caenorhabditis elegans. Nematologica 1987;24:63-71.

Stiernagle T. Maintenance of C. elegans. WormBook 2006:1-11.

Sulston JE. Post-embryonic development in the ventral cord of Caenorhabditis elegans. Philos Trans R Soc Lond B Biol Sci 1976;275:287-97.

Sulston JE, Horvitz HR. Post-embryonic cell lineages of the nematode, Caenorhabditis elegans. Dev Biol 1977;56:110-56.

White JG, Albertson DG, Anness MA. Connectivity changes in a class of motoneurone during the development of a nematode. Nature 1978;271:764-6.

Wicks SR, Roehrig CJ, Rankin CH. A dynamic network simulation of the nematode tap withdrawal circuit: predictions concerning synaptic function using behavioral criteria. J Neurosci 1996;16:4017-31. 